\title{
Le spectre identitaire
}

Entre langue et pouvoir au Mali

\section{Andrée Tabouret-Keller}

\section{(2) OpenEdition}

\section{Journals}

Édition électronique

URL : http://journals.openedition.org/esp/2812

DOI : 10.4000/esp.2812

ISSN : 2532-0319

Éditeur

Centre d'Information sur l'Éducation Bilingue et Plurilingue

Édition imprimée

Date de publication : 1 décembre 2013

Pagination : $91-94$

ISSN : 1127-266X

Référence électronique

Andrée Tabouret-Keller, «Le spectre identitaire », Éducation et sociétés plurilingues [En ligne], 35 | 2013, mis en ligne le 01 février 2020, consulté le 17 février 2021. URL : http://journals.openedition.org/esp/ 2812 ; DOI : https://doi.org/10.4000/esp.2812 


\section{COMPTES RENDUS D'OUVRAGES}

Wilma TONETTA, Gabriella VERNETTO, L'épreuve de français à l'examen d'État à la fin du cycle primaire en Vallée d'Aoste, Assessorat de l'Éducation et de la Culture, Département Surintendance des écoles, Aoste, septembre 2009.

Marie-Thérèse WEBER

Ce volume, issu des travaux de l'unité Résultats de l'apprentissage de la Structure régionale d'évaluation du système scolaire, analyse des épreuves de français d'élèves à la fin du premier cycle.

Avant l'autonomie de la vallée d'Aoste, des programmes ministériels s'appuyant sur des indications méthodologiques précises réglementaient l'école. Dès que l'autonomie a été accordée, «les équilibres entre l'Administration centrale et les écoles pour ce qui est de la planification didactique ont complètement changé» ( $\mathrm{p}$. 9). De ce fait, les indications de l'Administration centrale ne concernent plus, actuellement, la méthodologie didactique, mais les acquis des élèves lors des examens d'État du $1^{\mathrm{er}} \mathrm{du}$ du $2^{\text {ème }}$ cycle. La Vallée d'Aoste a eu «l'obligation de se donner les instruments juridiques et pédagogiques pour parvenir à des mesures d'accompagnement des écoles d'abord, d'harmonisation des critères d'évaluation ensuite et à des épreuves uniques sur tout le territoire régional à plus long terme» (p. 9). En 2000, une loi régionale «a prévu la constitution d'une structure régionale chargée de l'évaluation du système valdôtain» (p. 9). Cette structure, qui a vu le jour en 2007, est soutenue et contrôlée par un Comité scientifique et un Comité consultatif.

L'étude présentée par W. Tonetta et G. Vernetto a été élaborée pour aider les enseignants impliqués dans la préparation des épreuves de français à l'Examen d'Etat de fin de cycle. Elle vise «à harmoniser le format et l'évaluation des épreuves avec le Cadre européen commun de référence pour les langues: Apprendre, Enseigner, Évaluer du Conseil de l'Europe» (p. 11).

\section{Cécile GANUT, Le spectre identitaire. Entre langue et pouvoir au Mali, 2008, Limoges, Lambert-Lucas \\ Andrée TABOURET-KELLER}

L'hypothèse générale qui oriente les recherches que rapporte cet ouvrage - et donc l'analyse de leurs résultats - est celle de l'hétérogénéité constitutive du langage à partir de laquelle se construi- 
sent les processus d'homogénéisation d'une langue; ceux-ci ont comme supports les discours politiques et scientifiques qui visent à réduire l'hétérogénéité à des formes normatives explicites qui fonctionnent comme autant de mises en frontières aboutissant à des «agencements collectifs d'énoncés, plus ou moins figés, au sein des continuums langagiers» (p. 15).

L'hypothèse est mise à l'épreuve au Mali que l'auteur re-situe dans le cadre linguistique complexe de l'Afrique noire. Elle est introduite et discutée dans l'Introduction «Pour une anthropologie des pratiques langagières» dans lequel l'auteur précise les conditions exceptionnelles de son travail: le partage à Bamako, parfois pendant de longues périodes, de la vie d'une grande famille, une quinzaine de personnes, avec laquelle se nouent depuis 1993 des liens d'amitié. Ce «terrain»- pour reprendre les termes de l'auteur - est l'occasion de «conversations libres» enregistrées à propos des pratiques langagières avec différents membres de la famille, leur analyse va conduire à préciser comment les processus d'homogénéisation prolifèrent constamment sur le fond de l'hétérogénéité constitutive.

Dès le premier chapitre de l'ouvrage, «Des hommes qui parlent», l'appui constant sur des transcriptions d'enregistrements permet à l'auteur d'illustrer comment «la multiplicité des usages dans un même lieu et pour un même locuteur [l'a conduite] à appréhender les paroles dans leur hétérogénéité même» (p. 19). Les discours épilinguistiques des locuteurs sur les pratiques de langage sont révélateurs des processus de subjectivation à l'œuvre dans leur prise de parole et des positionnements adoptés dans les interactions; leur analyse conduit à des interprétations qualitatives dont on ne peut postuler aucune généralisation dans la mesure où «pour chaque contexte interactionnel, des éléments comme la territorialisation, la mobilité, le parcours biographique, la place dans la filiation, le désir de ville, les relations sociales et personnelles avec l'interlocuteur, etc., peuvent jouer de manière complexe dans l'émergence des formes» (p. 20). Le parcours de ce chapitre est l'occasion de préciser l'incidence des positionnements subjectifs des locuteurs, en particulier dans le jeu des pratiques des cousinages à plaisanterie, face aussi aux déplacements multiples liés aux obligations domestiques et sociales et, de manière plus générale, à la mobilité des places avec son cortège d'identifications et de prises de distance. Dans ce cadre général, «la subjectivité se définie d'emblée dans l'entre-deux des noms, des places, des positionnements. Elle n'est pas une substance et se construit à tout moment» (p. 36). Pour autant, les locuteurs qui, pour mille rai- 
sons, nomment, catégorisent, mettent en frontières des groupes autant que des langues, produisent des agencements au quotidien, dans les actes autant que par la parole, qui font osciller en permanence ces frontières (p. 49). Et ce malgré les facilités offertes par le discours courant à l'étayage de ce que l'auteur nomme «les fantasmes de l'UN », parmi lesquels elle retient la nomination, la catégorisation et la hiérarchisation (p. 50). Parmi de multiples exemples tirés des entretiens, l'auteur choisit le cas de «la langue malinké», aujourd'hui inscrite dans la liste des langues nationales du Mali, pour rétablir le déséquilibre qui l'avait écartée au profit du bambara. Si le locuteur ordinaire «navigue dans de multiples formes bamakoises, sans se préoccuper de savoir ce qu'elles représentent 'culturellement' ou 'identitairement', il reste que la part des discours précédents, qui pour des raisons politiques évidentes ont conservé leur attrait, est toujours sous-jacente ils 'proviennent d'un autre continent, l'Europe, auquel l'Afrique a été fortement liée lors des colonisations'» (p. 60).

«La mise en discours de l'Afrique» - le titre du second chapitre présente un vaste historique des écrits sur l'origine des langues et sur les langues en Afrique, de J. Herder avec le Traité sur l'origine des langues (1770) à J.- L. Amselle et M'Bokolo avec Au coeur de l'ethnie. Ethnie, tribalisme et État en Afrique (1995, ré.éd. 1999), avec un large arrêt sur les écrits de M. Delafosse, entre 1912 et 1929, et ses classifications ethniques fondées sur des critères linguistiques, en particulier entre malinké et bambara. Les sciences du langage actuelles ne sont pas en reste dans cette mise en discours: le français devient-il aujourd'hui une langue africaine et la sociolinguistique se met-elle au service de la francophonie? (p. 91).

Les graves questions soulevées au chapitre 2. trouvent des illustrations concrètes au cours du chapitre 3. «L'invention des langues» avec de riches extraits d'entretiens, la plupart réalisés à Bamako (Mali). Le colonialisme, avec les hiérarchisations des langues qu'il a produites et imposées - langues ethniques, langues riches, langues pauvres - l'ethnologie avec les états de choses et les énoncés qu'elle met en place, laissent des traces qui persistent mais qui révèlent les changements en cours: par exemple, l'opposition entre le malinké et le bambara encore attestée dans les discours, ne l'est plus du tout dans les pratiques. Le modèle de «l'état-nation», le langage politique qu'il génère, ne manquent pas de retentir dans la manière dont les populations d'origine rurale tentent de s'insérer dans la vie urbaine tout en se raccrochant à des identités auxquelles ils demandent de sauvegarder leurs origines - c'est le versant traditionaliste - et de promouvoir leurs nouvelles insertions - 
c'est le versant internationaliste. Pendant ce temps, le français conserve une suprématie que la pratique d'Internet, en cours d'extension, conforte.

Qu'apporte à cette situation déjà bien complexe le cadrage politique de l'instrumentalisation des langues par l'État? c'est la question à laquelle le chapitre 4. «L'instrumentalisation politique des langues» tente de répondre. Le français demeure la langue officielle du Mali qui à ce jour compte treize langues nationales qu'il convient de gérer. Les grands secteurs où l'État devrait, voire doit intervenir sont ceux de l'école et de la radio où les directives en faveur des langues nationales rencontrent de solides résistances. Par exemple, les élèves des écoles bilingues qui associent une langue nationale au français obtiennent, à l'entrée du second cycle des résultats bien inférieurs $(29,56 \%)$ que ceux des élèves monolingues en français $(41,66 \%)$; les écoles bilingues passent pour des écoles de paysans, sans avenir (p. 145). Du côté de la radio, si du point de vue linguistique le bambara est langue dominante, tout est fait pour veiller à la représentation des différentes cultures du pays grâce à des pratiques culturelles diverses comme les chants et les danses traditionnels. Dans le même temps, la pratique des cousinages à plaisanterie qui était un des piliers de la cohésion sociale, est affectée par l'urbanisation, les cousinages deviennent plus lâches cédant du terrain aux relations qui se construisent en fonction des nouvelles réalités politiques. Où placer alors un avenir possible pour un pouvoir authentiquement africain? le mythe d'une langue à soi continue à être entretenu comme garante du «génie de l'âme noire» (p. 166), le patrimoine linguistique à sauver nourrissant le fantasme d'origine des «afrocentristes» par l'écriture dans une langue africaine (plutôt qu'en français).

Dans ses conclusions, Entre langue et pouvoir, Cécile Canut envisage la possibilité d'une pluralité de langues et du multiculturalisme qui garantirait à chacun sa liberté et sa singularité. C'est dans les milieux urbains des jeunes, plus qu'ailleurs, que la multiplicité des formes d'expression, «la grande liberté des écritures et des traductions, le flou permanent entre écrit et oral attestent de manière exemplaire l'hétérogénéité des pratiques langagières» (p. 206). 